\title{
Myelinated Skin Sensory Neurons Project Extensively throughout Adult Mouse Substantia Gelatinosa
}

\author{
M. Danilo Boada and C. Jeffery Woodbury \\ Department of Zoology and Physiology, University of Wyoming, Laramie, Wyoming 82071
}

\begin{abstract}
The substantia gelatinosa (SG) of the dorsal horn of the spinal cord is a recipient zone for unmyelinated sensory neurons in adults. Recent studies of the central anatomy of physiologically identified skin sensory neurons in neonatal mice have shown that this region also receives substantial inputs from a variety of myelinated afferents. The present experiments were performed to determine whether these neonatal inputs represent a transient phenotype that retracts from the SG. Studies were conducted in an in vivo spinal cord preparation from adult mice; thoracic levels were targeted to facilitate comparisons with previous in vitro findings. We show that the SG continues to receive substantial projections from myelinated skin sensory neurons throughout life. A large population of myelinated nociceptors conducting in the upper $\mathrm{A} \delta$ and low $\mathrm{A} \beta$ range maintained extensive projections throughout all areas of the SG well into adulthood; the latter gave rise to dorsally recurving "flame"-shaped arbors extending into the marginal layer that were identical to afferents described in neonates and after nerve injury in adult rats. Furthermore, exquisitely sensitive down hair follicle afferents projected throughout the inner half of the SG (i.e., lamina IIi) and sent dense clusters of terminals well into the outer SG (IIo), where they intermingled with those of unmyelinated nociceptors. Arguments are presented that the SG likely plays a predominant role in tactile processing under normal conditions, but that this role switches rapidly to nociceptive-only during environmental exigencies imposed by temperature extremes.
\end{abstract}

Key words: mouse; skin; sensory neurons; nociceptors; superficial dorsal horn; tactile

\section{Introduction}

The substantia gelatinosa (SG), also known as lamina II, is a distinctive translucent region capping the dorsal horn of the spinal gray matter. Defined by its lack of myelin, the SG is generally equated with unmyelinated sensory inputs, which have been shown to terminate in this region (Sugiura et al., 1986). Less widely appreciated, however, is the fact that the SG also receives direct inputs from myelinated skin sensory neurons. Indeed, down hair afferents have been shown to project into the ventral SG (Light and Perl, 1979), and, using a semi-intact ex vivo preparation from neonatal mice, this adult organization was found to develop shortly after birth (Woodbury et al., 2001). Additional studies using this in vitro preparation have also shown that myelinated nociceptors project extensively throughout the SG in neonates (Woodbury and Koerber, 2003). The fate of these inputs from myelinated nociceptors, however, remains unclear.

Studies of the central projections of putative myelinated afferents labeled with cholera toxin conjugates argue that such inputs to the SG are no longer present in adults (for review, see Fitzgerald, 2005), in accord with the absence of myelin in this region. If true, this would predict that the growth of myelinated nociceptor central projections is initially exuberant and these arbors subse-

Received 0ct. 6, 2007; revised Jan. 13, 2008; accepted Jan. 13, 2008.

This work was supported by National Institutes of Health Grants NS44094 (C.J.W.) and P20 RR15640. We thank R. Carroll for help with animal husbandry and D. S. Woodbury for excellent technical assistance.

Correspondence should be addressed to C. Jeffery Woodbury, Department of Zoology and Physiology, University

of Wyoming, Laramie, WY 82071. E-mail: woodbury@uwyo.edu.

DOI:10.1523/JNEUROSC1.5609-07.2008

Copyright $\odot 2008$ Society for Neuroscience $\quad 0270-6474 / 08 / 282006-09 \$ 15.00 / 0$ quently undergo extensive pruning during early postnatal life or, alternatively, that the cells responsible for these widespread SG projections represent a transient population that is lost through cell death postnatally. However, uncertainty surrounds the identities of afferents that transport cholera toxin (Tong et al., 1999; Woodbury et al., 2001; Sántha and Jancsó, 2003), and bulklabeling studies may lack sufficient power to resolve this important question regarding SG functional organization (Woodbury et al., 2000, 2001; Woodbury and Koerber, 2003).

We therefore sought to resolve this issue through anatomical analyses of individual, physiologically identified afferents to the SG in adults. Mice were used because the short distances allow terminals to be labeled intracellularly from DRG somata, providing access to all afferent classes. However, to obviate potential problems surrounding the viability of central terminals under prolonged in vitro conditions, we developed a new in vivo spinal cord/DRG preparation for these purposes. Studies were conducted at thoracic levels to facilitate comparison with earlier in vitro findings from the same body region in neonates.

A previous report examined the physiological properties of mouse skin sensory neurons in vivo (Boada and Woodbury, 2007). Here we show that myelinated nociceptors continue to project extensively throughout the SG in adulthood, and that these projections are identical to similar afferents in neonates. Thus, as shown previously for tactile afferents, the present studies reveal that the central terminations of myelinated nociceptors are adult-like shortly after birth, and do not retract as predicted on the basis of bulk-labeling studies. Furthermore, down hair follicle afferents (D-HFAs) project more extensively throughout the SG 
than previously recognized, providing a major tactile input to a region historically equated with nociception and pain.

\section{Materials and Methods}

All procedures used in the present experiments were approved by the Institutional Animal Care and Use Committee of the University of Wyoming and conform to National Institutes of Health guidelines. The in vivo mouse thoracic preparation used in the present experiments has been described in detail (Boada and Woodbury, 2007). Briefly, adult Swiss-Webster mice of either sex (4-10 weeks of age, 25-40 g) were anesthetized with ketamine and xylazine $(90$ and $10 \mathrm{mg} / \mathrm{kg}$, respectively, i.p.) or sodium pentobarbital ( $75 \mathrm{mg} / \mathrm{kg}$, i.p.). Hair over the head, neck, and ipsilateral trunk was clipped. The trachea was cannulated for ventilation with humidified oxygen, and the cerebral cortex was exposed and aspirated full thickness before immobilization with D-tubocurarine (Sigma, St. Louis, MO); EKG, carotid pressure, and end-tidal $\mathrm{CO}_{2}$ were monitored and maintained within normal limits throughout all surgical procedures, electrophysiological recordings, and subsequent survival periods required for intracellular labeling with Neurobiotin (NB).

A dorsal midline incision was made in trunk skin, and DRGs at thoracic levels T11/12 were exposed by laminectomy. Desiccation was prevented by continuous superfusion with oxygenated artificial CSF (in mM: 127.0 NaCl, $1.9 \mathrm{KCl}, 1.2 \mathrm{KH}_{2} \mathrm{PO}_{4}, 1.3 \mathrm{MgSO}_{4}, 2.4 \mathrm{CaCl}_{2}, 26.0 \mathrm{NaHCO}_{3}$, and 10.0 D-glucose) flowing through an in-line heater (MPRE8; Cell MicroControls, Norfolk, VA). The spinal column was secured with custom clamps and the preparation then transferred to a heated recording chamber. Skin, rectal, and DRG temperatures, monitored with calibrated thermocouples, were maintained at $32 \pm 1^{\circ} \mathrm{C}, 35 \pm 1^{\circ} \mathrm{C}$, and $37 \pm 0.5^{\circ} \mathrm{C}$, respectively, as described (Boada and Woodbury, 2007).

Electrophysiology. DRG neuronal somata were impaled with quartz micropipettes (80-150 M $\Omega$ ) containing 20\% NB (Vector Laboratories, Burlingame, CA) in $1 \mathrm{~m}$ potassium acetate, and stable penetrations with a resting membrane potential less than or equal to $-45 \mathrm{mV}$ were studied further. Recordings were digitized for subsequent off-line analyses using Spike2 (Cambridge Electronic Design, Cambridge, UK).

Receptive fields (RFs) were located with the aid of a stereomicroscope using a standard mechanical search sequence; the latter progressed from light touch with a fine sable hair paintbrush to searching with blunt probes and ultimately gentle to noxious pinch with fine-tipped forceps. RFs were found across the entire dermatome in these intact preparations, from dorsal to ventral midlines; those located near the incision site were excluded. Afferents were identified as cutaneous if RFs tracked with skin displacement; afferents for which a mechanically sensitive RF could not be found were not studied further. Mechanical thresholds were determined with calibrated von Frey filaments (Stoelting, Wood Dale, IL). Adaptation rate was evaluated using suprathreshold probes mounted in a micromanipulator; skin stretch and vibratory stimuli were also tested in many cells.

Thermal stimuli were delivered using a $3 \times 5 \mathrm{~mm}$ feedback-controlled Peltier stimulator (Yale Instrument Repair and Design Shop, New Haven, CT); to displace insulating air and standardize heat transfer, a droplet of water was placed on the RF, and the temperature of the water interface was monitored with a second calibrated thermocouple to measure the actual temperature experienced by the epidermal surface. To eliminate the potential for spurious mechanical stimulation by the thermode during changes in temperature, the thermode was occasionally withdrawn slightly to leave only water in contact. Areas of skin exposed to heat were monitored, and cells recorded subsequently with overlapping RFs were rejected.

After characterization with natural stimuli, a bipolar stimulating electrode was brought into contact with the RF for measurements of electrically evoked spike latency $(50-100 \mu \mathrm{s}, 0.5 \mathrm{~Hz})$. Latency measurements were obtained using the minimum intensity required to excite neurons consistently without jitter; utilization time was not taken into account. To allow calculation of conduction velocity, conduction distances were measured for each afferent after termination of the experiment by careful dissection and measurement of the distance from the RF to the center of the DRG along the closest nerve.

Neuronal labeling. After physiological characterization, selected neu- rons were iontophoretically injected with Neurobiotin (+1.5-4 nA, 75\% duty cycle, $10-68 \mathrm{nA} \cdot \min$ total). The number of cells stained per DRG was $\leq 2$ and depended on adequate dorsoventral separation within the dermatome. Five to twenty-two hours later, animals were heparinized and transcardially perfused with saline followed by either $4 \%$ paraformaldehyde (PFA) or 3\% PFA and 1\% glutaraldehyde in $0.1 \mathrm{M} \mathrm{PBS,} \mathrm{pH}$ 7.3. Most post-iontophoretic survival times were $\leq 9 \mathrm{~h}$, as these were found to be sufficient to label equivalent numbers of central collaterals as longer survival periods (e.g., overnight) within the length of spinal cord (four consecutive segments) analyzed in these experiments. Fixed spinal cords were dissected free and blocked, embedded in $10 \%$ gelatin, postfixed in PFA, and cryoprotected in $20 \%$ sucrose. Frozen transverse sections $(50 \mu \mathrm{m})$ were serially collected in PBS and reacted with standard ABC-DAB techniques (Elite kit; Vector Laboratories) to visualize NB as detailed previously (Woodbury et al., 2001); in some cases, serial sections were cut with a vibratome and permeabilized with ethanol before ABC$\mathrm{DAB}$ processing. Sections were rinsed, mounted, and minimally air dried to affix to slides. To minimize distortion of superficial dorsal horn in anatomical analyses as a result of shrinkage artifacts, sections were rehydrated and temporarily coverslipped in PBS. Labeled arbors were then serially reconstructed in hydrated sections at $200-1000 \times$, photographed, and drawn with the aid of a camera lucida. Digital photomicrographs were obtained using a Retiga Exi (QImaging, Surrey, British Columbia, Canada) and IPLab (Scanalytics, Rockville, MD); images were minimally manipulated using Adobe Photoshop (Adobe Systems, San Jose, CA) to normalize brightness and contrast. After documentation, coverslips were removed, and sections were then dehydrated, cleared, and permanently coverslipped. Arbors drawn and/or photographed in hydrated sections were checked against the same arbors in cleared tissue sections to verify completeness of documentation. All values are presented as mean $\pm \mathrm{SD}$.

\section{Results}

The present report is based on observations on 57 individual physiologically identified cutaneous primary afferents that were well labeled in adult mice in vivo. These represent a restricted sample from a larger population $(>450)$ of skin sensory neurons that have been physiologically identified and characterized in adult mice in vivo. Because neurons were intracellularly recorded and stained from their somata, the central anatomy of a broad spectrum of afferents could be examined, including 24 myelinated tactile afferents, 20 myelinated nociceptors, and 13 unmyelinated (C-fiber) nociceptors.

Emphasis in the current report is placed on myelinated cutaneous afferents with projections into the SG; as noted (see Materials and Methods) (see also Boada and Woodbury, 2007), only afferents with mechanically sensitive cutaneous RFs were included in these studies. To provide a reference for comparative purposes, the locations of unmyelinated nociceptors and myelinated tactile afferent terminations are described. In addition, a novel population of myelinated nociceptors that predominantly terminate ventral to the SG is described.

\section{Unmyelinated (C-fiber) afferents}

In accord with seminal in vivo studies in guinea pig (Sugiura et al., 1986) and in vitro studies in mice (Woodbury et al., 2001, 2004; Albers et al., 2006), all labeled C-nociceptors terminated in the SG and, to a lesser extent, marginal zone (lamina I). Most (12/13) were $\mathrm{C}$-polymodal nociceptors that responded to moderately firm stroking of the RF with a fine paintbrush (mechanical threshold, $0.63 \pm 0.29 \mathrm{mN}$; range, $0.16-1.24 \mathrm{mN}$ ) as well as heat (threshold, $42.2 \pm 3.2^{\circ} \mathrm{C}$; range, $38-46^{\circ} \mathrm{C}$ ) and cold (threshold, $16.3 \pm 4.5^{\circ} \mathrm{C}$; range, $10-21^{\circ} \mathrm{C}$ ). The remaining C-nociceptor was a high-threshold mechanoreceptor (C-HTMR) that responded only to intense mechanical stimuli (i.e., skin pinch with forceps). 

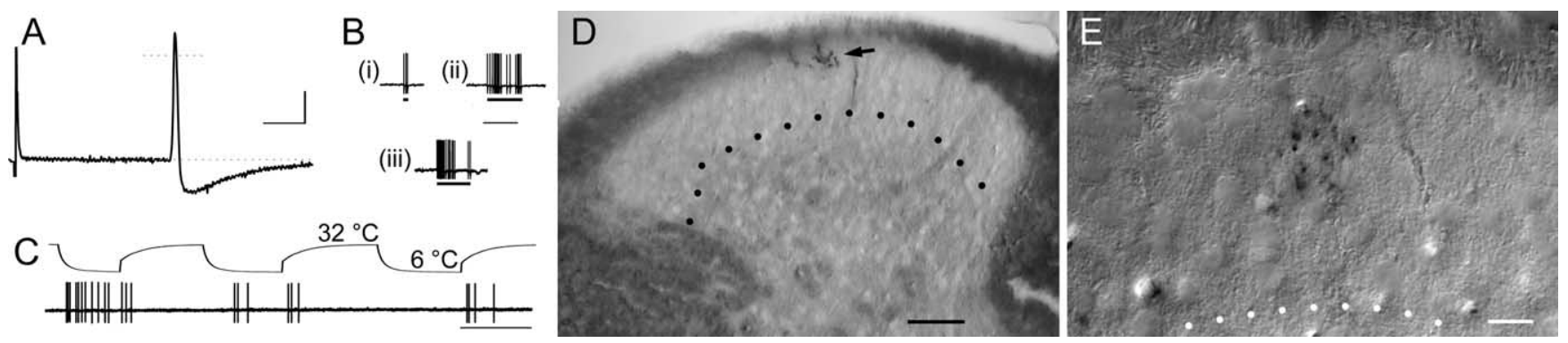

Figure 1. Unmyelinated (C) nociceptors terminate in outer SG. Examples from two C-polymodal nociceptors are shown in $\boldsymbol{A}-\boldsymbol{D}$ and $\boldsymbol{E}$. Note the well circumscribed arborizations in lamina Ilo, evident at low- $(\boldsymbol{D}$, arrow) and high- $(\boldsymbol{E})$ power photomicrographs; in this and all subsequent figures, dotted lines indicate the ventral border of the SG, and midline is to the right. Stereotypical physiological properties are exemplified in $\boldsymbol{A}-\boldsymbol{C}$; cells exhibited broad somal spikes with long AHPs $(\boldsymbol{A})$, relatively low mechanical thresholds $(\boldsymbol{B})$, sensitivity to cooling $(\boldsymbol{C})$, and robust activation by heat (data not shown). Bars under mechanically evoked responses $(\boldsymbol{B})$ signify $0.35 \mathrm{mN}(\boldsymbol{i}), 1.3 \mathrm{mN}(\boldsymbol{i i})$, and stimulation with a fine paintbrush (iii). Note that although these afferents habituated rapidly to repeated cooling, rebound responses after termination of cooling did not. Postnatal ages, CVs, and mechanical thresholds of afferents in $A-D$ and $\boldsymbol{E}$ were $40 \mathrm{~d}, 0.7 \mathrm{~m} / \mathrm{s}$, and $0.76 \mathrm{mN}$ and $32 \mathrm{~d}, 0.8 \mathrm{~m} / \mathrm{s}$, and $0.35 \mathrm{mN}$, respectively. Physiology calibration: $\boldsymbol{A}, 20 \mathrm{mV} ; \boldsymbol{B}, \boldsymbol{C}, 80 \mathrm{mV} ; \boldsymbol{A}, 10 \mathrm{~ms} ; \boldsymbol{B}, 2 \mathrm{~s} ; \boldsymbol{C}, 20 \mathrm{~s}$. Scale bars: $\boldsymbol{D}, 50 \mu \mathrm{m} ; \boldsymbol{E}, 10 \mu \mathrm{m}$.

Representative central projections from C-polymodal nociceptors stained in adult mice in vivo are shown in Figure 1; those from the C-HTMR were essentially identical (data not shown). Arbors gave rise to a longitudinal column of dense terminal neuropil that was well circumscribed in the transverse plane and extended uninterrupted over many sections $(400-550 \mu \mathrm{m})$ in the rostrocaudal plane. As seen in Figure 1, terminal arbors were centered in the outer SG (i.e., lamina IIo), with only minimal overlap into lamina I; occasional processes extended ventrally into laminas IIi and III as noted previously (Sugiura et al., 1986; Sugiura, 1996; Woodbury et al., 2001).

\section{Myelinated skin sensory neurons \\ Tactile afferents}

Well labeled central projections from a variety of tactile afferents have been recovered, including guard hair follicle $(n=6)$, slowly adapting type I (SAI; $n=4)$ and type II $(n=2)$, Pacinian $(n=3)$, mechano/cold $(n=4)$, and D-hair follicle afferents $(n=5)$; general physiological properties of these afferents were described previously (Boada and Woodbury, 2007). With the exception of D-HFAs (see below), the central arbors of tactile afferents generally remained deep to the SG (e.g., Fig. 6G) and were identical in all major respects to published descriptions of the same afferent populations in mice (Woodbury et al., 2001; Koerber and Woodbury, 2002; Woodbury and Koerber, 2007) and cats (for review, see Brown, 1981); the central anatomy of mechano/cold afferents is the subject of a separate report (M. D. Boada and C. J. Woodbury, unpublished observations).

\section{D-hair follicle afferents}

Every well labeled D-HFA gave rise to substantial inputs into the SG as exemplified in Figure 2. All exhibited exquisite mechanical sensitivity (thresholds $<0.07 \mathrm{mN}$ ) that was rivaled only by SAI afferents. The conduction velocities (CVs) of those labeled ranged from 1.53 to $14 \mathrm{~m} / \mathrm{s}$; three of the five stained (conducting at $1.53,1.8$, and $2.3 \mathrm{~m} / \mathrm{s}$ ) were only slightly above the A/C-fiber cutoff of $1.5 \mathrm{~m} / \mathrm{s}$ (Fig. 2A-D) (Boada and Woodbury, 2007), whereas one conducted in the low $\mathrm{A} \beta$ range (average, $4.99 \pm 5.26$ $\mathrm{m} / \mathrm{s}$; median, $2.3 \mathrm{~m} / \mathrm{s}$ ). Thus, these afferents exhibited a broader $\mathrm{CV}$ range than typically reported, and in the absence of information on central anatomy (below), those with the most slowly conducting axons could easily be mistaken for unmyelinated (C) low-threshold mechanoreceptors. Unlike the latter $(n=2$; not labeled) (Boada and Woodbury, 2007), all cells classified as D-HFAs responded equally well to both fast- and slow-moving stimuli and were silent during cooling (data not shown) (for
C-LTMR cooling responses, see Albers et al., 2006) when care was taken to prevent skin movement.

As seen in Figure 2, the central anatomy of D-HFAs was unique and highly stereotypical. Central axons ascended and descended in the medial dorsal column and gave rise to dorsally recurving, "flame-shaped" collateral arbors that supported dense clusters of large, bouton-like varicosities extending well into the SG. Multiple (7-16) well labeled collaterals were recovered from each afferent in our sample. As with other types of cutaneous sensory neurons (Koerber and Mirnics, 1995; Wilson et al., 1996; Woodbury et al., 2001), considerable variability was observed between individual collateral arbors along the rostrocaudal axis.

Representative examples of the most complex and superficially projecting arbors are shown in Figure 2. The latter were located in the main projection zone containing the greatest arborization density. Importantly, individual collateral arbors in this region overlapped extensively along the rostrocaudal axis to form uninterrupted columns of longitudinally oriented neuropil as documented in neonates [Woodbury et al. (2001), their Fig. 8]. Because of this overlap, the rostrocaudal extent of individual D-HFA collateral arbors could not be determined. Nevertheless, the longitudinal spread of individual arbors in this region was substantial, particularly evident where collaterals arose at widely spaced intervals. For example, the arborization illustrated in Figure $2 B-D$ was part of an uninterrupted longitudinal column that extended beyond the immediately adjacent collateral axons located $750 \mu \mathrm{m}$ rostrally and $200 \mu \mathrm{m}$ caudally (data not shown). Thus, although this columnar organization characterizing the main termination zone of D-HFAs was similar to that formed by C-polymodal nociceptors (above), the length of these D-hair columns more than doubled that of the columns formed by C-polymodal nociceptors. This columnar organization of D-HFA central projections was not observed in earlier studies using horseradish peroxidase (HRP) (Light and Perl, 1979; Hayashi, 1985), presumably reflecting limited diffusion of the latter relative to NB (Koerber and Mirnics, 1995; Wilson et al., 1996).

As in neonates, these continuous columns of neuropil formed by adult D-HFAs occupied the inner half of the SG (i.e., lamina IIi). Unlike neonates, however, adult arbors in this main central projection extended more dorsally, well into the outer half of the SG (i.e., lamina IIo), occasionally extending all the way to the border with the marginal layer (Fig. 2). Importantly, in all D-HFAs labeled, this main central projection zone was invariably bracketed by isolated (i.e., nonoverlapping) collateral arbors that remained deeper and exhibited a simpler morphology as docu- 

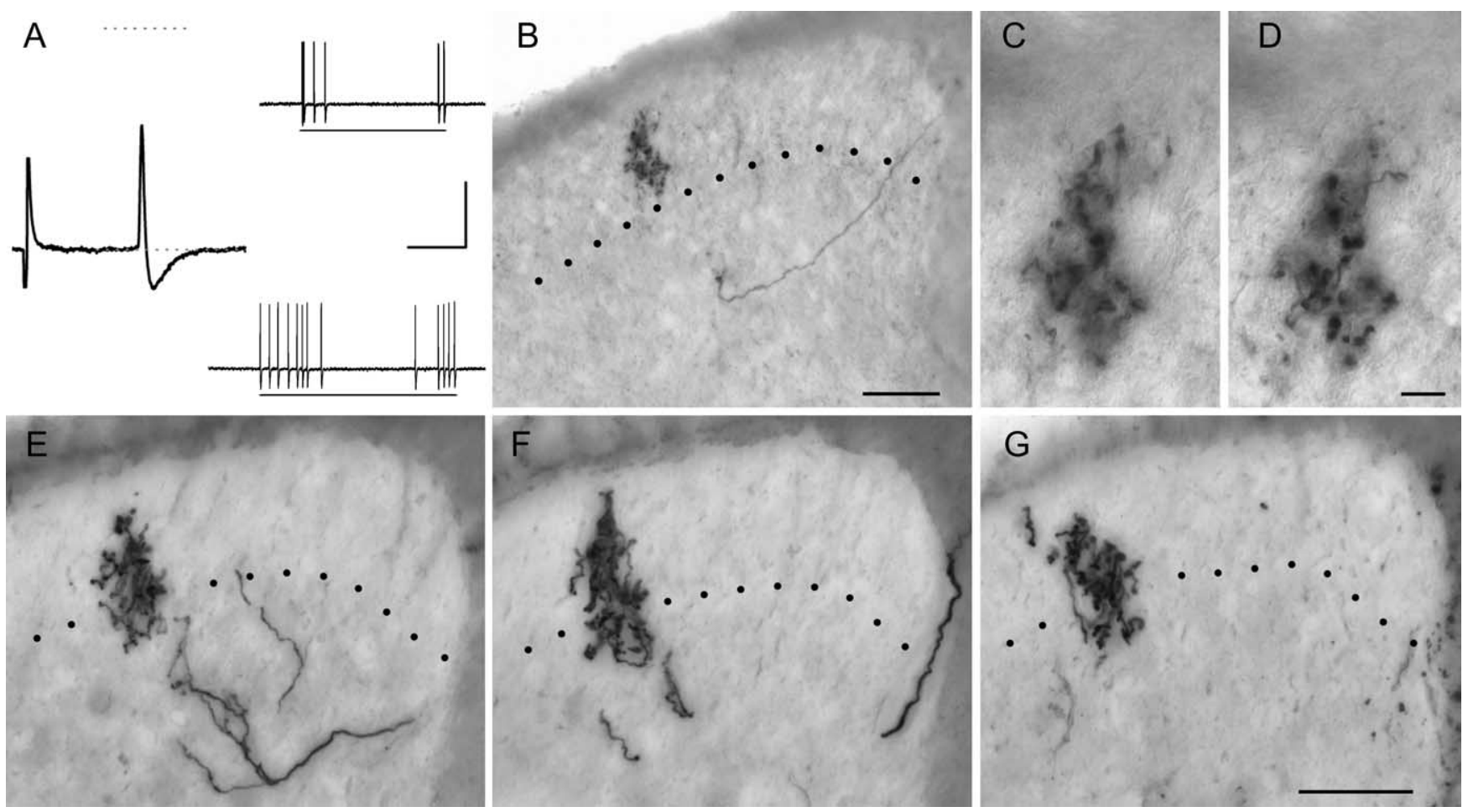

Figure 2. D-hair follicle afferents terminate throughout the SG. Examples from two different D-HFAs are shown in $\mathbf{A}-\mathbf{D}$ and $\boldsymbol{E}-\mathbf{G}$. Characteristically brief somal action potentials and rapidly adapting response properties of these afferents are exemplified in $A$; bars below mechanically evoked responses represent $0.07 \mathrm{mN}$ (top right) and light touch with a mounted probe (bottom right). Note the substantial $S G$ input from these sensitive afferents, evident at low $(\boldsymbol{B})$ and higher $(\boldsymbol{C}, \boldsymbol{D})$ power, with multiple large boutons located in both inner and outer $\mathbf{S G}$; the afferent in $\boldsymbol{E}-\mathbf{G}$ abutted the ventral border of the marginal zone $(\boldsymbol{F}) \cdot \boldsymbol{B}-\boldsymbol{D}$, Single focal plane photomicrographs. $\boldsymbol{E}-\mathbf{G}$, Composite photomicrographs from multiple planes through tissue sections. Postnatal ages, $C V s$, and mechanical thresholds of afferents in $\boldsymbol{A}-\boldsymbol{D}$ and $\boldsymbol{E}-\boldsymbol{G}$ were $40 \mathrm{~d}, 1.8 \mathrm{~m} / \mathrm{s}$, and $<0.07 \mathrm{mN}$ and $45 \mathrm{~d}, 5.3 \mathrm{~m} / \mathrm{s}$, and $<0.07 \mathrm{mN}$, respectively. Calibration: $20 \mathrm{mV}$; spike, $10 \mathrm{~ms}$; evoked responses, $250 \mathrm{~ms}$. Scale bars: $\boldsymbol{B}, \mathbf{E}-\mathbf{G}, 50 \mu \mathrm{m} ; \boldsymbol{C}, \boldsymbol{D}, 10 \mu \mathrm{m}$.

mented for other tactile afferents (Koerber and Mirnics, 1995; Wilson et al., 1996; Woodbury et al., 2001); the latter outliers arborized primarily in the dorsal nucleus proprius (i.e., lamina III) with only minor overlap into the ventral SG (i.e., lamina IIi) as seen in the small numbers of isolated D-HFA collaterals labeled in seminal HRP studies (Light and Perl, 1979).

\section{Nociceptors}

Myelinated nociceptors that were well labeled in the present study $(n=20)$ spanned a broad range of peripheral CVs, from 3.5 to $31 \mathrm{~m} / \mathrm{s}$ (average, $9.07 \pm 6.17 \mathrm{~m} / \mathrm{s}$; median, $6.9 \mathrm{~m} / \mathrm{s}$ ). Five of those labeled exhibited CVs $>11 \mathrm{~m} / \mathrm{s}$, the $\mathrm{A} \beta / \delta$ cutoff at this core temperature (Boada and Woodbury, 2007). Most RFs could not be located with a fine paintbrush; although probing with sharptipped forceps was generally effective, many required skin pinch for activation. Some exhibited relatively low thresholds, whereas others could not be induced to respond to the stiffest von Frey filaments tested (e.g., $100 \mathrm{mN}$ ), with the result that mechanical thresholds spanned at least three orders of magnitude $(0.07-100.1 \mathrm{mN})$. All exhibited narrow, uninflected somal action potentials, and all but one exhibited long-duration afterhyperpolarizations (AHPs) with remarkably slow recovery kinetics, a characteristic of myelinated nociceptors at normal physiological temperatures (Boada and Woodbury, 2007). A broad diversity of central morphology was observed, roughly divisible into three basic groups as described below.

Lamina I-V nociceptors. The majority of myelinated nociceptors labeled in the present studies $(n=13)$ gave rise to dorsally recurving, "flame-shaped" collateral arbors that extended throughout laminas I-V. These were identical to a subset that was previously noted in young mice (Woodbury and Koerber, 2003). Representative examples of this anatomically distinctive class labeled in adults in vivo are shown in Figures 3 and 4. Although most of these afferents exhibited CVs $<11 \mathrm{~m} / \mathrm{s}$ (i.e., A $\delta$ peripheral axons), three conducted in the low $A \beta$ range $(12-13 \mathrm{~m} / \mathrm{s})$, and thus this nociceptor class is not defined by CV categories based on compound action potentials (Boada and Woodbury, 2007).

Lamina I-V nociceptors exhibited moderately high mechanical thresholds (average, $3.23 \pm 2.52 \mathrm{mN}$; range, $0.33-9.02 \mathrm{mN}$; median, $3.4 \mathrm{mN}$ ). No inverse correlation between threshold and CV was evident, as both high and low mechanical thresholds were distributed throughout the CV spectrum (data not shown); the latter suggests that this group in adults, as in neonates (Woodbury and Koerber, 2003), encompasses more than one of the subcategories identified originally (Burgess and Perl, 1967). All responded to increasing forces with an increasingly vigorous, slowly adapting discharge. As noted previously in neonates, the pattern of discharge to maintained suprathreshold stimuli was often highly regular, similar to the type II pattern defined previously (Chambers et al., 1972). Most RFs were punctate, although multiple sensitive foci could be discerned on occasion.

As in neonates, the central arborizations of these afferents were relatively diffuse, even in the main central projection zone containing the most complex arbors. Nevertheless, as illustrated in Figure 4, these fibers gave rise to substantial arborization in the dorsal horn, with well labeled examples distributing 8-12 collaterals from both rostrally and caudally directed parent axons in the dorsal columns. As with D-HFAs (above), the arbors of individual collaterals exhibited considerable rostrocaudal overlap 


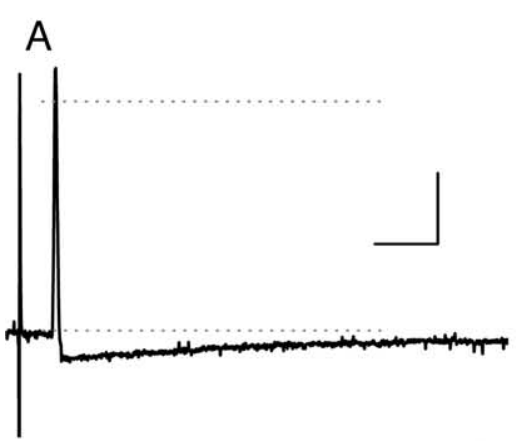

B

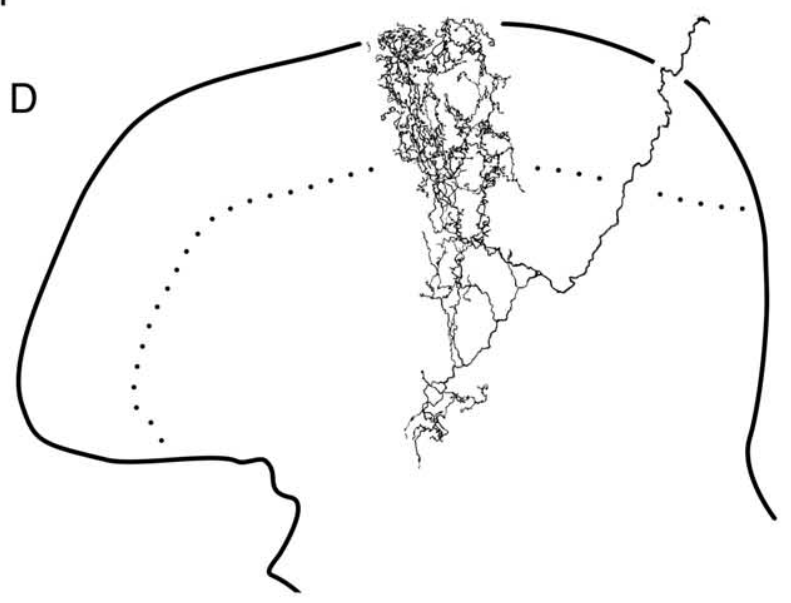

F

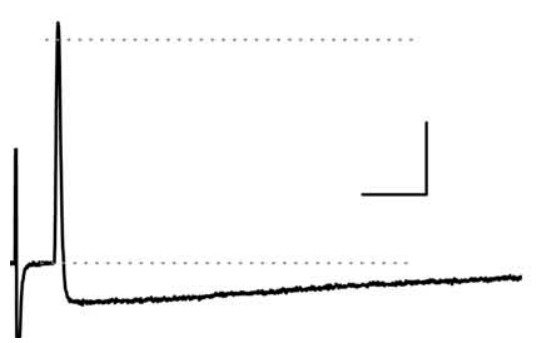

G

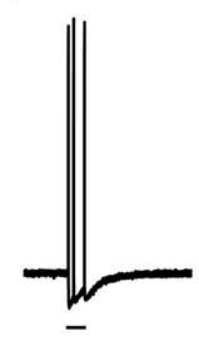

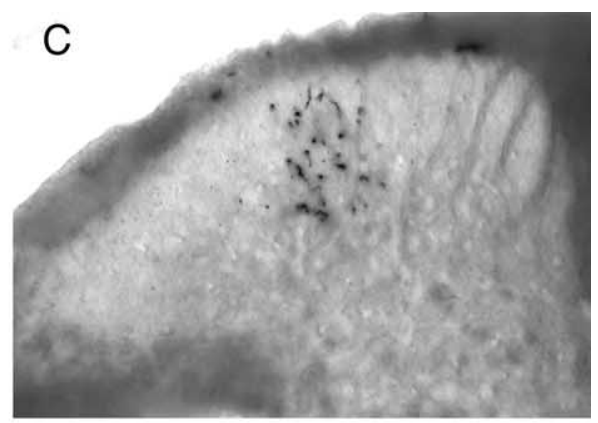
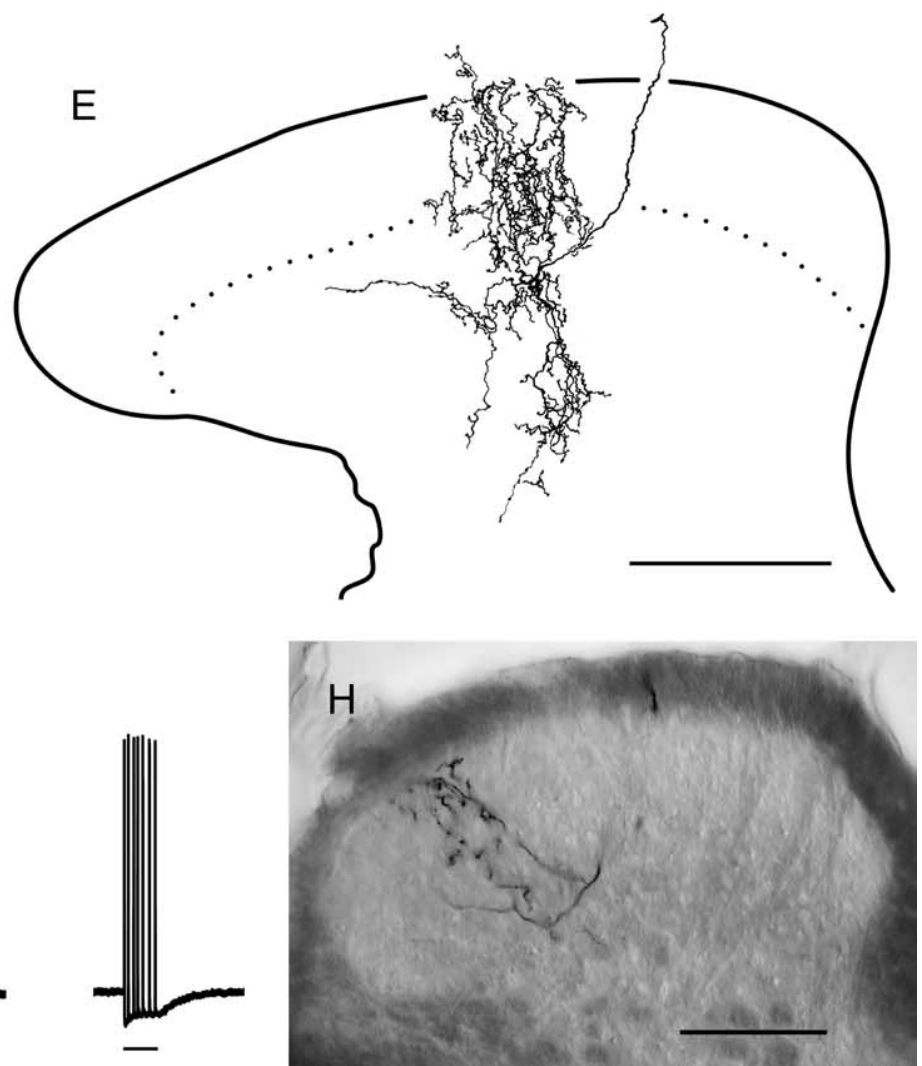

Figure 3. Most myelinated nociceptors terminate throughout the SG. Examples from two different lamina I-V myelinated nociceptors are shown in $\boldsymbol{A}-\boldsymbol{E}$ and $\boldsymbol{F}-\boldsymbol{H}$ (the latter is also shown in Fig. 4). $\boldsymbol{A}, \boldsymbol{B}, \boldsymbol{F}, \boldsymbol{G}$, Action potentials $(\boldsymbol{A}, \boldsymbol{F})$ and mechanically evoked responses $(\boldsymbol{B}, \boldsymbol{G})$ of afferents shown in $\boldsymbol{C}-\boldsymbol{E}$ and $\boldsymbol{H}$, respectively. The bars under evoked responses in $\boldsymbol{B}$ signify (from left to right) 5.3 and $9.2 \mathrm{mN}$ (3.4 and $5.3 \mathrm{mN}$ in $\boldsymbol{G}$ ). Note the dorsally recurving "flame-shaped" arbors of these afferents, extending throughout the dorsal horn with extensive arborization throughout superficial laminas ( $\boldsymbol{C}-\boldsymbol{E}, \boldsymbol{H}$; see also Fig. 4). The photomicrograph in $\boldsymbol{C}$ was taken midway between two collateral branch points. Ages, $\mathrm{CV}$, and mechanical thresholds of afferents in $\boldsymbol{A}-\boldsymbol{E}$ and $\boldsymbol{F}-\boldsymbol{H}$ were $35 \mathrm{~d}$, $6.0 \mathrm{~m} / \mathrm{s}$, and $0.35 \mathrm{mN}$ and $43 \mathrm{~d}, 5.8 \mathrm{~m} / \mathrm{s}$, and $0.76 \mathrm{mN}$, respectively. Neither responded to cooling or heating stimuli (data not shown). Calibration bars: $20 \mathrm{mV} ; \boldsymbol{A}, \boldsymbol{F}, 10 \mathrm{~ms} ; \boldsymbol{B}, \boldsymbol{G}, 500 \mathrm{~ms}$. Scale bars: $\boldsymbol{E}($ for $\boldsymbol{D}, \boldsymbol{E}), \boldsymbol{H}($ for $\boldsymbol{C}, \boldsymbol{H}), 100 \mu \mathrm{m}$.

throughout the main projection (e.g., see Fig. 4 legend), whereas isolated collateral arbors located rostral and caudal to this region exhibited a much simpler morphology.

Although collaterals arborized throughout all dorsal horn laminas, the density of arborization in deeper laminas (i.e., IV and V) was comparatively minor; the majority of arborization, particularly in the main projection zone, was centered on more dorsal laminas (i.e., I-III). At high magnification, small, relatively widely spaced en passant varicosities were evident throughout the arborization. Processes ascending to lamina I frequently changed course to run longitudinally in this lamina as in neonates. Importantly, identical findings were observed between afferents recovered from mice that varied widely in age [postnatal day 31 (P31)P68]; thus, this distinctive central arborization pattern is ontogenetically stable and does not retract or disappear in adults as argued on the basis of bulk labeling (Fitzgerald, 2005).
Lamina I/IIo nociceptors. Only two myelinated nociceptors in our sample exhibited the classical central morphology normally associated with myelinated nociceptors (Light and Perl, 1979), with central axons in Lissaur's tract and horizontally oriented arborizations predominantly centered on lamina I but also extending into the outer SG (lamina IIo; not shown); as in neonates (Woodbury and Koerber, 2003), these afferents in adult mice also gave rise to occasional processes extending deeper (laminas IIi and occasionally III). As with most lamina I-V nociceptors (above), CVs of this small sample fell within the A $\delta$ range (5.7 and $10.3 \mathrm{~m} / \mathrm{s}$, respectively), and mechanical thresholds varied widely; for example, one equaled the lowest value seen among lamina I-V nociceptors $(0.33 \mathrm{mN}$; above), whereas the other required skin pinch for activation; thus, this distinctive morphological subtype also appears to encompass more than one of the originally identified subcategories (Burgess and Perl, 1967). 

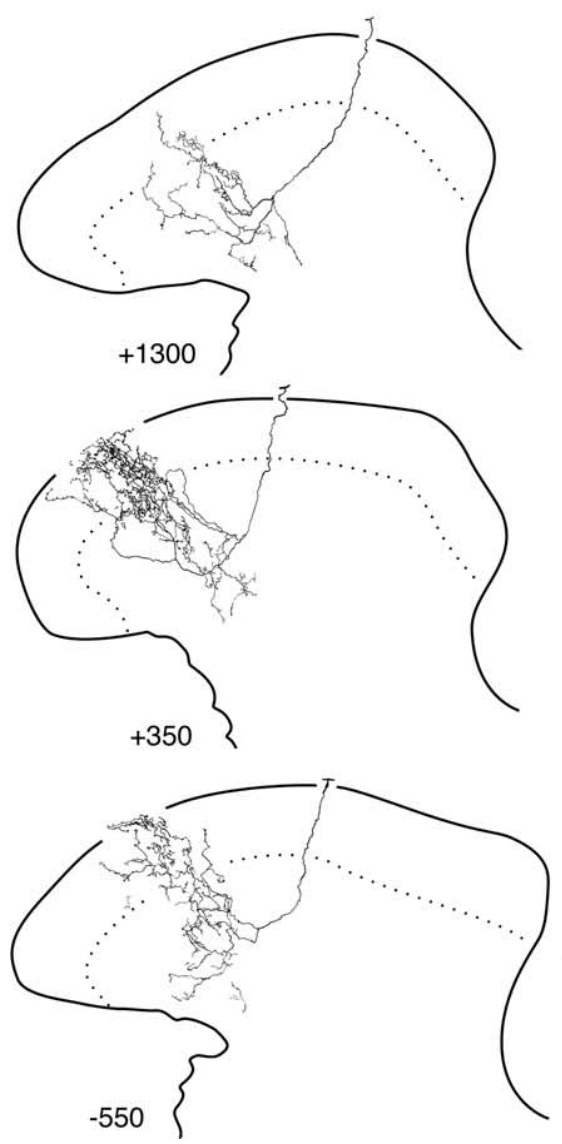

Figure 4. Myelinated nociceptors give rise to extensive inputs to the dorsal horn. Shown are camera lucida reconstructions (4-5 $50 \mu \mathrm{m}$ sections each) of all collateral arborizations recovered from the afferent in Figure 3H; numbers represent distance of collateral branch points (in micrometers) from the first collateral rostral to the bifurcation of the central axon after root entry (designated " 0 "). Essentially uninterrupted labeling was seen between the collateral arbors designated " +700 " and " -550 "; hence, reconstructions in this primary projection zone were arbitrarily limited rostrocaudally to contain $200-250 \mu \mathrm{m}$ of tissue. Scale bar, $100 \mu \mathrm{m}$.

"Deep dorsal horn" nociceptors. Five myelinated nociceptors were found to exhibit an unexpected central morphology, with dorsally recurving collateral arborizations restricted to relatively deep dorsal horn locations. Arbors from two of these afferents extended into the ventral SG (i.e., lamina IIi), although none projected into classical nocireceptive regions (i.e., laminas IIo or I). Although this sample is somewhat heterogeneous, all "deep" nociceptors are treated together because of their small number.

This subset spanned a broad range of CVs $(4.7-31 \mathrm{~m} / \mathrm{s})$, with the majority ( 3 of 5 ) conducting in the $\mathrm{A} \beta$ range (average, $14.6 \pm$ $10.0 \mathrm{~m} / \mathrm{s}$; median, $12 \mathrm{~m} / \mathrm{s}$ ). An example of a "deep" A $\beta$ nociceptor with inputs to the ventral SG is shown in Figure 5. The latter was one of only two myelinated nociceptors encountered to date in our in vivo studies that responded to noxious heat [out of $>50$ tested to $53-55^{\circ} \mathrm{C}$ (Boada and Woodbury, 2007, unpublished observations)]. This heat-sensitive nociceptor repeatedly responded with one spike to the finest von Frey filament, although it responded with an increasingly vigorous, slowly adapting discharge to increasing forces as with other myelinated nociceptors. Although the response to heat on first exposure was weak, sensitization to subsequent heating was evident (Fig. 5C). Centrally, a total of eight well labeled collateral arbors were recovered; reconstructions of four of the most elaborate arbors located in the region of greatest overlap are shown in Figure 5, D and E. Arbors appeared more diffuse than typically seen among lamina I-V nociceptors (above); furthermore, they were predominantly restricted to laminas III-V, with relatively minor extension into lamina IIi.
Three other "deep" nociceptors gave rise to arbors that remained deep to the $S G$, including the fastest myelinated nociceptor encountered; the two most elaborate arbors of the latter are illustrated in Figure 6. The mechanical threshold of this fast $A \beta$ nociceptor was identical to that exhibited by another "lamina III-V" nociceptor conducting in the A $\delta$ range, whereas the other A $\delta$ example (Fig. $6 E$ ) required skin pinch for activation. Thus, as in other myelinated nociceptor subtypes (above), this anatomically defined sample likely includes multiple subtypes that were identified originally (Burgess and Perl, 1967). Interestingly, the central arbors of these nociceptors were often highly reminiscent of those from SAIs (Fig. 6G) (Koerber and Woodbury, 2002; Woodbury and Koerber, 2007), which cautions against inferring function on the basis of laminar termination patterns.

\section{Discussion}

The present study examined the central anatomy of individual, physiologically identified cutaneous afferents that were intracellularly labeled with NB in adult mice in vivo. The results of these studies provide a number of fundamental insights into the organization of tactile and nociceptive inputs to the superficial dorsal horn. First, despite its apparent lack of myelin, the SG receives far more extensive inputs from myelinated afferents than previously documented; this, coupled with the fact that the SG receives major inputs from D-HFAs (below), cautions against equating this region with unmyelinated inputs in general and/or nociceptive processing in particular. Second, the novel central morphology of 


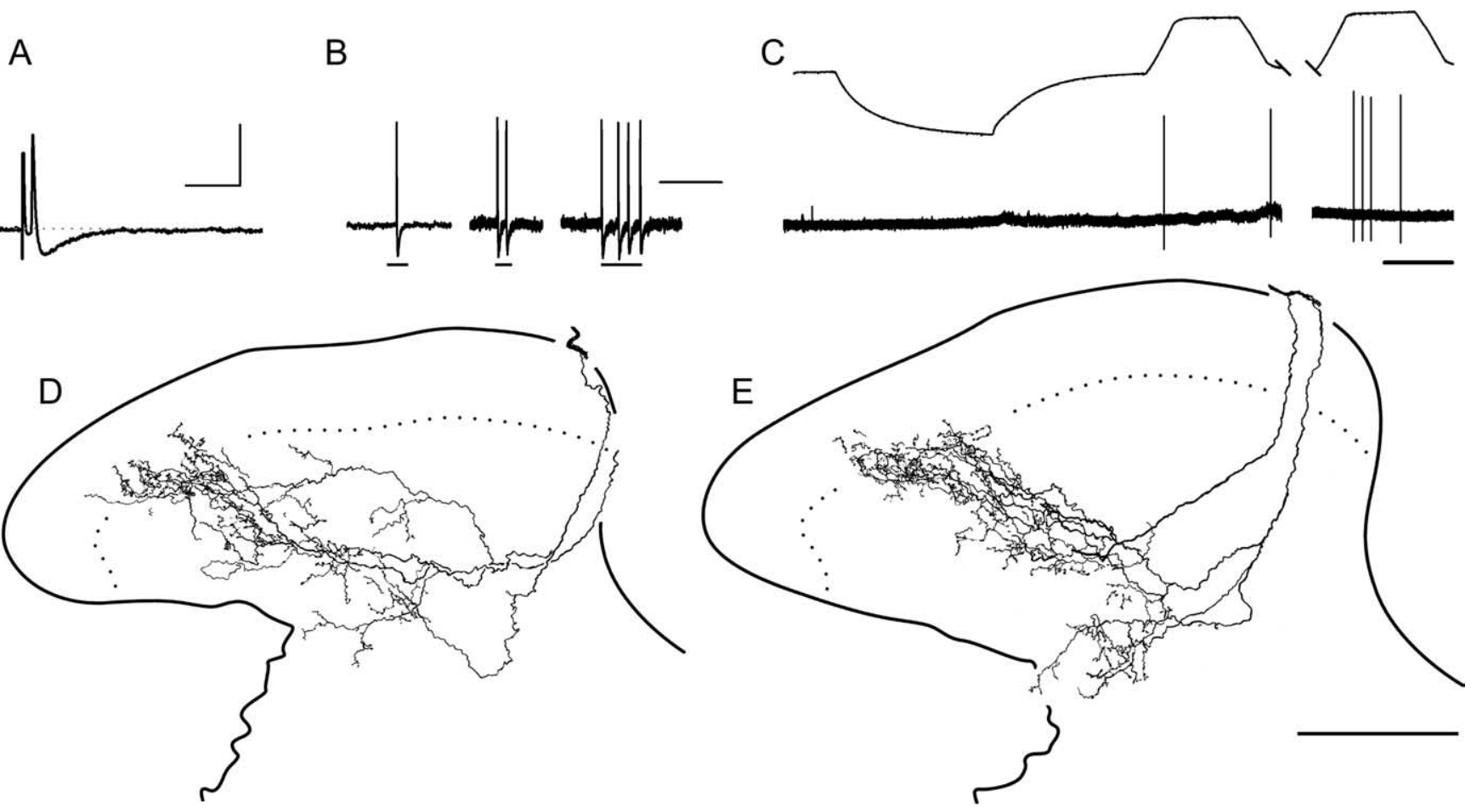

Figure 5. Physiological and anatomical properties of an A $\beta$ polymodal nociceptor. Relatively short-duration AHP (A) was atypical for myelinated nociceptors (compare with Figs. 3, 6). Furthermore, this afferent was relatively sensitive to low mechanical forces $(\boldsymbol{B}$; bars from left to right signify $0.07,0.16$, and $0.35 \mathrm{mN})$. Sensitization to repeated heating is evident in $\boldsymbol{C}$. $\boldsymbol{D}, \boldsymbol{E}$, Note that arbors extended dorsally into ventral SG. Postnatal age, $\mathrm{CV}$, mechanical threshold, and heat threshold (first exposure) were $47 \mathrm{~d}, 16 \mathrm{~m} / \mathrm{s}, 0.07 \mathrm{mN}$, and $53^{\circ} \mathrm{C}$, respectively. Calibration: $20 \mathrm{mV}$; $A, 10$ $\mathrm{ms} ; \boldsymbol{B}, 100 \mathrm{~ms} ; \boldsymbol{C}, 10$ s. Scale bar, $100 \mu \mathrm{m}$.

A

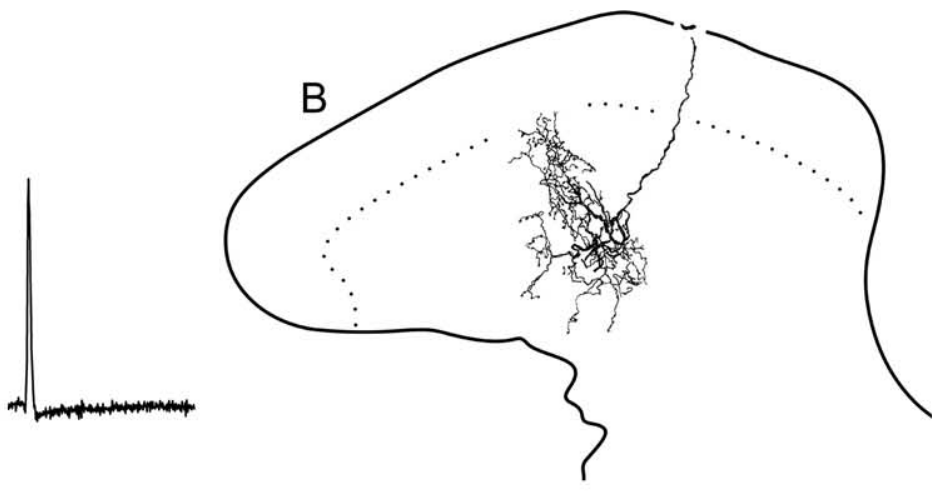

D

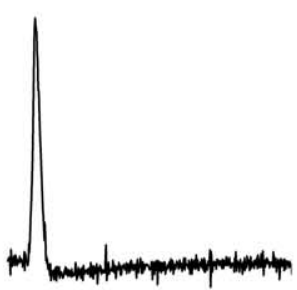

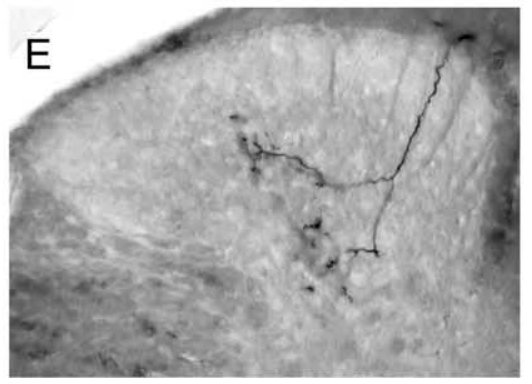

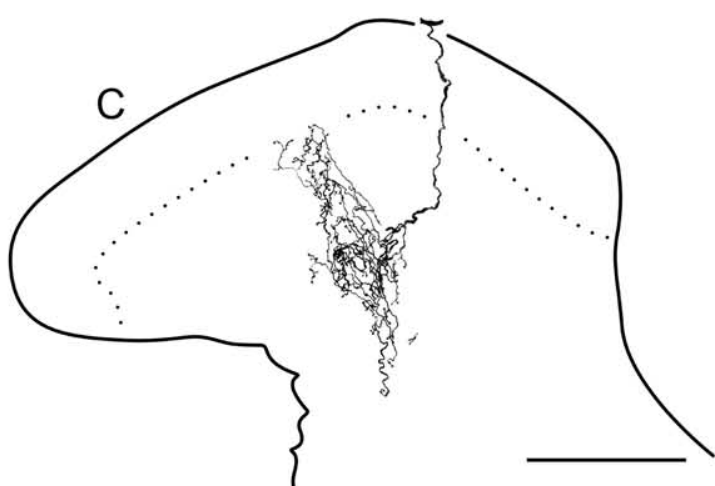

$\mathrm{F}$

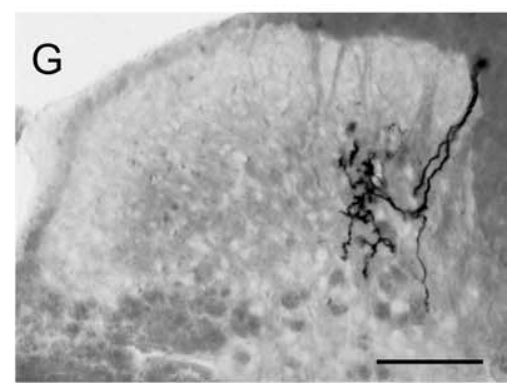

Figure 6. Some myelinated nociceptors terminate deep to the SG. Somal spike and central arbors of the fastest conducting myelinated nociceptor encountered (CV $=31 \mathrm{~m} / \mathrm{s}$ ) are shown in $\boldsymbol{A}-\boldsymbol{C}$; those of an $A \delta$ nociceptor ( $C V=4.8 \mathrm{~m} / \mathrm{s}$ ) that required pinch for activation are shown in $\boldsymbol{D}$ and $\boldsymbol{E}$. Note apparent similarities in central anatomy to that of $S A I$ afferents ( $\boldsymbol{G}$; spike of the latter shown in $\boldsymbol{F}$ ). Postnatal age and mechanical threshold of $A \beta$ nociceptor were $39 \mathrm{~d}$ and $0.16 \mathrm{mN}$, respectively; neither responded to noxious thermal stimuli (data not shown). Calibration: $20 \mathrm{mV}, 5 \mathrm{~ms}$. Scale bars, $100 \mu \mathrm{m}$.

myelinated nociceptors described previously in neonates (Woodbury and Koerber, 2003) represents a miniaturized replica of normal adult anatomy. That is, the extensive projections throughout the SG from neonatal myelinated nociceptors un- dergo little if any change throughout postnatal life; importantly, they do not retract from this region as suggested on the basis of bulk-transport studies. Finally, multiple types of myelinated nociceptors can be discriminated on the basis of central anatomy; 
the majority represent continua that are not bounded by classical definitions based on CV or mechanical threshold.

\section{A $\boldsymbol{\beta}$ nociceptors}

As the sensory neurons underlying first pain (Campbell and LaMotte, 1983), myelinated nociceptors occupy an important component of central mechanisms that have evolved to safeguard the integument. In view of their importance, remarkably few studies have examined the central termination patterns of myelinated nociceptors in adults (Light and Perl, 1979; Hayashi, 1985). Furthermore, as previous studies were focused on slowly conducting examples, the central terminations of fast-conducting myelinated nociceptors have remained obscure. The present studies suggest there is nothing unique about the central morphology of $\mathrm{A} \beta$ myelinated nociceptors. Those labeled simply represented fasterconducting versions of more slowly conducting counterparts, with one possible exception. To date, lamina I/IIo-specific myelinated nociceptors with $\mathrm{A} \beta \mathrm{CV}$ s have not been observed. However, the fastest-conducting example was very near the $A \beta / \delta$ cutoff, and thus a larger sample of this relatively rare subtype may also reveal a continuum spanning classical CV boundaries. Interestingly, a subset of nociceptors, including the fastest encountered, projected only to the nucleus proprius, a region typically associated with tactile afferents. Whether their terminals segregate specifically to wide dynamic range and/or nociceptive-specific interneurons in this region (Zhang and Giesler, 2006; Mazarío and Basbaum, 2007) remains to be determined.

\section{Lamina I-V nociceptors}

Myelinated nociceptors with dorsally recurving "flame-shaped" arborizations throughout laminas I-V represented the most common type encountered in these studies. A few exhibited peripheral CVs in the low $\mathrm{A} \beta$ range, and although similar nociceptors arborizing throughout all laminas have not yet been documented outside mice, this distinctive central anatomy exhibits some similarity to a subset of incompletely labeled nociceptors in cat and monkey (Light and Perl, 1979; Rethelyi et al., 1982) and is identical to that of a few unidentified $\mathrm{A} \beta$ fibers labeled after nerve injury in rats (Woolf et al., 1992). Importantly, the present findings reveal that this morphologically unique class of afferents is not only present but arborizes extensively throughout the dorsal horn of normal (i.e., uninjured) adult mice and may account for earlier observations of occasional $\mathrm{A} \beta$ inputs to marginal neurons in primates (Kumazawa and Perl, 1978).

Given their existence in normal adults, it is unclear why the projections from these myelinated nociceptors throughout the SG have escaped notice in bulk-transport studies using cholera toxin-binding subunit. The latter preferentially binds myelinated cutaneous afferents in rodents and has been widely used in studies of nerve central labeling patterns in normal animals (for review, see Woodbury et al., 2001). Because labeling is observed in lamina I after cholera toxin transport (Woodbury et al., 2000), it is unlikely that myelinated nociceptors simply fail to transport this marker. A more likely explanation is that bulk-transport techniques simply lack sufficient sensitivity for visualization of these diffuse inputs.

\section{Substantia gelatinosa: pleasure or pain?}

The superficial dorsal horn in general, and SG in particular, is commonly equated with nociception and pain. Although many nociceptors project selectively to this region, this generalization does not apply to most myelinated nociceptors (e.g., laminas $\mathrm{I}-\mathrm{V})$, and some avoid it entirely. Furthermore, the present studies highlight the fact that the SG of mice also receives substantial inputs from D-HFAs, among the most exquisitely sensitive tactile afferents that innervate the body surface; a rare population of unmyelinated tactile afferents also project to this region (Sugiura et al., 1986; Sugiura, 1996). Importantly, D-HFAs were found to project far more dorsally than previously documented in lamina IIi (Woodbury et al., 2001) [see also Light and Perl (1979), Rethelyi et al. (1982), and Hayashi (1985)], well into lamina IIo, where they were clearly in position to intermingle with unmyelinated nociceptor terminals. This suggests that D-hair inputs are not yet fully mature in neonates (cf. Woodbury et al., 2001), growing farther into the SG during postnatal life. As this appears to significantly alter current understanding of afferent inputs to adult SG, potential reasons for this discrepancy with earlier studies warrant consideration.

One possibility is that this more superficial termination of D-HFAs is more apparent than real, because the SG appears to be thicker in mice than in larger species (see discussion in Woodbury et al., 2000); thus, whereas a ventral expansion of this region may explain a relatively more dorsal termination of mouse D-HFAs overall, it cannot explain D-hair arbors abutting the lamina I/II border (see Results). A related possibility is that this more superficial termination reflects significant species and/or segmental differences in D-HFAs themselves, as previous studies in adults were conducted at caudal levels in cat and monkey (Light and Perl, 1979) or cranial levels in cat (Hayashi, 1985). However, although this possibility cannot presently be ruled out given the lack of NB-based studies outside mice, it appears unlikely.

Despite differences in segmental level, no major differences in laminar termination patterns have been observed across diverse tactile afferent subclasses between mice and other species (present findings) [see also Woodbury et al. (2001), Koerber and Woodbury (2002), and Woodbury and Koerber (2003, 2007)]; hence, D-HFAs would appear to provide a unique exception. However, observations of isolated D-hair arbors invading "the deep two-thirds of lamina II" in the spinal trigeminal nucleus caudalis of cat (Hayashi, 1985) not only suggest otherwise, but may help explain the substantial portion of cat SG that remains unlabeled at spinal levels after isolectin- $\mathrm{B}_{4}$ transport (Woodbury et al., 2000). Moreover, a more superficial termination of tactile afferents than commonly held is supported by the finding that type II synaptic glomeruli, formed by D-HFAs (Rethelyi et al., 1982 ), constitute $\sim 20 \%$ of the glomerular structures in lamina IIo (Ribeiro-da-Silva and Coimbra, 1982). Thus, the most parsimonious explanation may simply reflect the fact that the use of $\mathrm{NB}$ in the present studies labeled greater numbers of collaterals in this diminutive species than previous attempts with HRP in larger species (Light and Perl, 1979; Hayashi, 1985), revealing both the most superficially terminating collaterals in the main central projection and deeper isolated outliers (Koerber and Mirnics, 1995; Wilson et al., 1996).

Importantly, because D-HFAs are activated by the gentlest breeze, far more tactile than nociceptive information would be expected to enter the SG on a daily basis, especially considering the sophisticated repertoire of protective behaviors working in concert to minimize nociceptor activation. The common tendency to equate the SG with nociception and pain therefore warrants reevaluation if the physiology of this region under normal conditions is to be accurately understood.

The function of D-HFA inputs to the SG is not yet clear. Although exquisitely sensitive $A \delta$ tactile afferents are present in human hairy skin nerves (Adriaensen et al., 1983) (but see Olausson et al., 2002; Cole et al., 2006), knowledge of their potential role in sensation 
is hampered by a lack of microstimulation studies. Nevertheless, substantial numbers of SG neurons respond well to light tactile stimuli, whether touch-specific or wide dynamic range neurons (Light et al., 1979; Bennett et al., 1980) (for review, see Light, 1992). Historically, while inhibition of nociceptive transmission by tactile inputs has received the most attention (Melzack and Wall, 1965) [see also Narikawa et al. (2000) and Lu and Perl (2003)], recent studies suggest that most SG circuits are excitatory (Lu and Perl, 2005; Santos et al., 2007) and that incoming sensory information is relayed to neurons outside the SG (Giesler et al., 1978; Willis et al., 1978; Light, 1992; Eckert et al., 2003).

Because tactile inputs are routinely and preferentially activated under normal conditions, it would appear that the SG normally plays a predominant role in tactile sensation, whether tickle (Light, 1992) or the pleasure of gentle caress (Olausson et al., 2002). Recent findings, however, reveal that all this may change instantly under noxious conditions, as D-HFAs quickly become mechanically inexcitable when their RFs are exposed to noxious temperatures (Woodbury and Boada, 2007), effectively silencing these inputs and thus allowing nociceptors uninhibited access to SG circuitry. This suggests, therefore, that the SG rapidly switches between opposing functional states in response to events occurring in the periphery.

\section{References}

Adriaensen H, Gybels J, Handwerker HO, Van Hees J (1983) Response properties of thin myelinated (A- $\delta$ ) fibers in human skin nerves. J Neurophysiol 49:111-122.

Albers KM, Woodbury CJ, Ritter AM, Davis BM, Koerber HR (2006) Glial cell line-derived neurotrophic factor expression in skin alters the mechanical sensitivity of cutaneous nociceptors. J Neurosci 26:2981-2990.

Bennett GJ, Abdelmoumene M, Hayashi H, Dubner R (1980) Physiology and morphology of substantia gelatinosa neurons intracellularly stained with horseradish peroxidase. J Comp Neurol 194:809-827.

Boada MD, Woodbury CJ (2007) Physiological properties of mouse skin sensory neurons recorded intracellularly in vivo: temperature effects on somal membrane properties. J Neurophysiol 98:668-680.

Brown AG (1981) Organisation in the spinal cord. New York: Springer.

Burgess PR, Perl ER (1967) Myelinated afferent fibres responding specifically to noxious stimulation of the skin. J Physiol (Lond) 190:541-562.

Campbell JN, LaMotte RH (1983) Latency to detection of first pain. Brain Res 266:203-208.

Chambers MR, Andres KH, von Duering M, Iggo A (1972) The structure and function of the slowly adapting type II mechanoreceptor in hairy skin. J Exp Physiol 57:417-445.

Cole J, Bushnell MC, McGlone F, Elam M, Lamarre Y, Vallbo A, Olausson H (2006) Unmyelinated tactile afferents underpin detection of low-force monofilaments. Muscle Nerve 34:105-107.

Eckert III WA, McNaughton KK, Light AR (2003) Morphology and axonal arborization of rat spinal inner lamina II neurons hyperpolarized by muopioid-selective agonists. J Comp Neurol 458:240-256.

Fitzgerald M (2005) The development of nociceptive circuits. Nat Rev Neurosci 6:507-520.

Giesler Jr GJ, Cannon JT, Urca G, Liebeskind JC (1978) Long ascending projections from substantia gelatinosa Rolandi and the subjacent dorsal horn in the rat. Science 202:984-986.

Hayashi H (1985) Morphology of terminations of small and large myelinated trigeminal primary afferent fibers in the cat. J Comp Neurol 240:71-89.

Koerber HR, Mirnics K (1995) Morphology of functional long-ranging primary afferent projections in the cat spinal cord. J Neurophysiol 74:2336-2348.

Koerber HR, Woodbury CJ (2002) Comprehensive phenotyping of sensory neurons using an ex vivo somatosensory system. Physiol Behav 77:589-594.

Kumazawa T, Perl ER (1978) Excitation of marginal and substantia gelatinosa neurons in the primate spinal cord: indications of their place in dorsal horn functional organization. J Comp Neurol 177:417-434.

Light AR (1992) The initial processing of pain and its descending control: spinal and trigeminal systems. In: Pain and headache, Vol 12 (Gildenberg PL, ed). Basal: Karger.
Light AR, Perl ER (1979) Spinal termination of functionally identified primary afferent neurons with slowly conducting myelinated fibers. J Comp Neurol 186:133-150.

Light AR, Trevino DL, Perl ER (1979) Morphological features of functionally defined neurons in the marginal zone and substantia gelatinosa of the spinal dorsal horn. J Comp Neurol 186:151-172.

Lu Y, Perl ER (2003) A specific inhibitory pathway between substantia gelatinosa neurons receiving direct C-fiber input. J Neurosci 23:8752-8758.

Lu Y, Perl ER (2005) Modular organization of excitatory circuits between neurons of the spinal superficial dorsal horn (laminae I and II). J Neurosci 25:3900-3907.

Mazarío J, Basbaum AI (2007) Contribution of substance P and neurokinin A to the differential injury-induced thermal and mechanical responsiveness of lamina I and V neurons. J Neurosci 27:762-770.

Melzack R, Wall PD (1965) Pain mechanisms: a new theory. Science 150:971-979.

Narikawa K, Furue H, Kumamoto E, Yoshimura M (2000) In vivo patchclamp analysis of IPSCs evoked in rat substantia gelatinosa neurons by cutaneous mechanical stimulation J Neurophysiol 84:2171-2174.

Olausson H, Lamarre Y, Backlund H, Morin C, Wallin BG, Starck G, Ekholm S, Strigo I, Worsley K, Vallbo ÅB, Bushnell MC (2002) Unmyelinated tactile afferents signal touch and project to insular cortex. Nat Neurosci 5:900-904.

Rethelyi M, Light AR, Perl ER (1982) Synaptic complexes formed by functionally defined primary afferent units with fine myelinated fibers J Comp Neurol 207:381-393.

Ribeiro-da-Silva A, Coimbra A (1982) Two types of synaptic glomeruli and their distribution in laminae I-III of the rat spinal cord. J Comp Neurol 209:176-186.

Sántha P, Jancsó G (2003) Transganglionic transport of choleragenoid by capsaicin-sensitive $\mathrm{C}$-fibre afferents to the substantia gelatinosa of the spinal dorsal horn after peripheral nerve section. Neuroscience 116:621-627.

Santos SFA, Rebelo S, Derkach VA, Safronov BV (2007) Excitatory interneurons dominate sensory processing in the spinal substantia gelatinosa of rat. J Physiol (Lond) 581:241-254.

Sugiura Y (1996) Spinal organization of C-fiber afferents related with nociception or non-nociception. Prog Brain Res 113:319-339.

Sugiura Y, Lee CL, Perl ER (1986) Central projections of identified, unmyelinated (C) afferent fibers innervating mammalian skin. Science 234:358-361.

Tong YG, Wang HF, Ju G, Grant G, Hokfelt T, Zhang X (1999) Increased uptake and transport of cholera toxin B-subunit in dorsal root ganglion neurons after peripheral axotomy: possible implications for sensory sprouting. J Comp Neurol 404:143-158.

Willis WD, Leonard RB, Kenshalo DRJ (1978) Spinothalamic tract neurons in the substantia gelatinosa. Science 202:986-988.

Wilson P, Kitchener PD, Snow PJ (1996) Intraaxonal injection of Neurobiotin reveals the long-ranging projections of $\mathrm{A} \beta$-hair follicle afferent fibers to the cat dorsal horn. J Neurophysiol 76:242-254.

Woodbury CJ, Boada MD (2007) Thermal filtering of tactile inputs: new hinge on an old gate. Soc Neurosci Abstr 33:124-127.

Woodbury CJ, Koerber HR (2003) Widespread projections from myelinated nociceptors throughout the substantia gelatinosa provide novel insights into neonatal hypersensitivity. J Neurosci 23:601-610.

Woodbury CJ, Koerber HR (2007) Central and peripheral anatomy of slowly adapting Type I low-threshold mechanoreceptors innervating trunk skin of neonatal mice. J Comp Neurol 505:547-561.

Woodbury CJ, Ritter AM, Koerber HR (2000) On the problem of lamination in the superficial dorsal horn of mammals: a reappraisal of the substantia gelatinosa in postnatal life. J Comp Neurol 417:88-102.

Woodbury CJ, Ritter AM, Koerber HR (2001) Central anatomy of individual rapidly adapting low-threshold mechanoreceptors innervating the "hairy" skin of newborn mice: early maturation of hair follicle afferents. J Comp Neurol 436:304-323.

Woodbury CJ, Zwick M, Wang S, Lawson JJ, Caterina MJ, Koltzenburg M, Albers KM, Koerber HR, Davis BM (2004) Nociceptors lacking TRPV1 and TRPV2 have normal heat responses. J Neurosci 24:6410-6415.

Woolf CJ, Shortland P, Coggeshall RE (1992) Peripheral nerve injury triggers central sprouting of myelinated afferents. Nature 355:75-78.

Zhang X, Giesler GJ (2006) Response characterstics of spinothalamic tract neurons that project to the posterior thalamus in rats. J Neurophysiol 93:2552-2564. 